\title{
Syntactic core composite sandwich (SCCS) with thermoplastic foam and expanded glass granulate core
}

\author{
Alexander Hackert ${ }^{1, a}$, Patrick Schaarschmidt ${ }^{2}$, Tristan Timmel $^{2}$ and Lothar Kroll ${ }^{1}$ \\ ${ }^{1}$ Institute of Lightweight Structures, Technische Universität Chemnitz, 09107 Chemnitz, Germany \\ ${ }^{2}$ Cluster of Excellence MERGE, Technische Universität Chemnitz, 09107 Chemnitz, Germany
}

\begin{abstract}
Ultra light, highly porous materials arranged as a sandwich structure with thermoplastic fiber reinforced composites (TP-FRC) as a cover layer have very good physical and mechanical properties. An essential prerequisite for the production of hybrid material composites is therefore the provision of material compatible and cost effective manufacturing processes. The lightweight potential of such a syntactic core composite sandwich (SCCS) with thermoplastic and expanded glass granules was examined with the aim of a mass production. After developing a manufacturing strategy, various samples are prepared and their mechanical properties are evaluated by performing bending tests and examination of micrographs.
\end{abstract}

\section{Introduction}

Increasing environmental and economic demands in mechanical engineering require specific material combinations and adjusted manufacturing processes. In mechanical engineering, the greatest potential for lightweight construction lies in the reduction of moving masses. Targeted lightweight construction allows the use of smaller drives and the design for lower forces. Thanks to lightweight composite materials, higher machining accuracy, speed, longer service life and lower material wear can be achieved. So it is possible in addition to the targeted adjustment of stiffness and strength, thoughtfully introduced residual stresses, to exploit the anisotropic strength behaviour and apply the special thermal properties. Due to the achievable synergy effects, especially in automotive lightweight design, multimaterial assemblies are increasingly applied. Therefore, carbon fibres reinforced plastics (CFRP) arranged with new isotropic syntactic foam structures to a Multimaterial Sandwich Composite (MSC) form the basis for novel weight-optimized as well as cost-effective applications. This leads to high specific stiffness and strength structures combined with excellent energy absorption capacity at high damage tolerances as required in a variety of technical applications.

\section{Manufacturing strategy}

A particular challenge is the processing of macroscopic expanded glass granules with grain sizes over $2 \mathrm{~mm}$ in a thermoplastic manufacturing process. The expanded glass (Figure 1) comes in the form of spherical granules with a grain density of $320 \mathrm{~kg} / \mathrm{m}^{3}$ and mean

\footnotetext{
a Corresponding author: alexander.hackert@mb.tu-chemnitz.de
}

grain strength of $1.4 \mathrm{~N} / \mathrm{mm}^{2}$. In addition, conventional PA6 granules in different degrees of filling are used for the thermoplastic matrix.
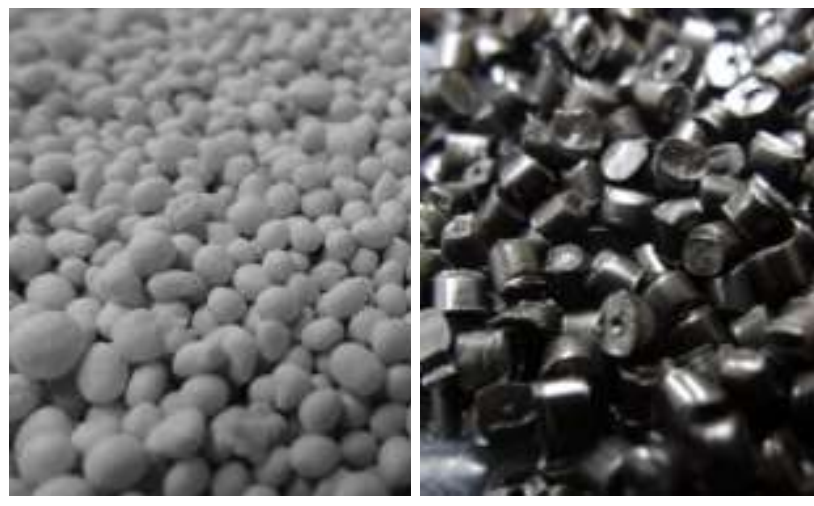

Figure 1. Expanded glass granules (left), conventional PA6 granules (right)

Due to the grain size, the processing in an injection moulding process is excluded. The expanded glass granules would be destroyed in the system and the high property potential would no longer be usable. Alternatively, the thermoplastic matrix material can be melted in a mould cavity and the expanded glass embedded. Therefore, a dipping edge tool with solid steel base plates that transfer heat evenly is used (Figure 2) in a heated press plant, which has two separately adjustable heating plates, which can each be heated up to $300^{\circ} \mathrm{C}$. The heating rates of the two plates are each a maximum of $15 \mathrm{~K} / \mathrm{min}$. With the cooling system connected in the test field can also be cooled at about $15 \mathrm{~K} / \mathrm{min}$. 


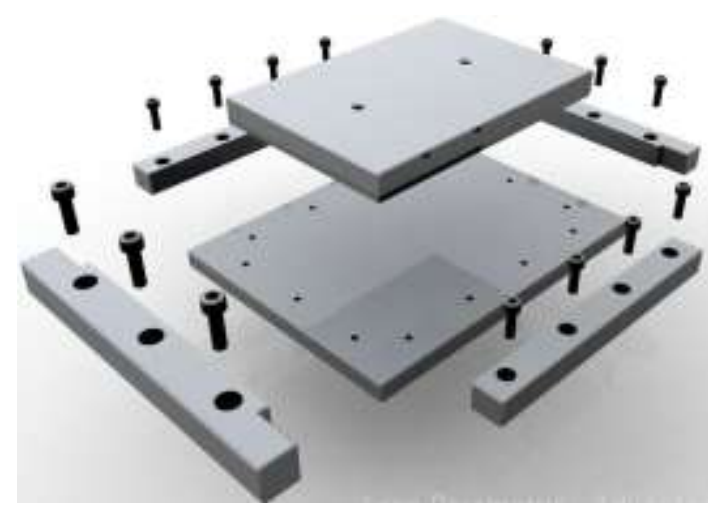

Figure 2. Thermal pressing tool in an exploded view

For the cover layers of the symmetric core composite unidirectional, carbon fibre reinforced, thermoplastic Prepregs are used. These thermoplastic semi-finished products pre-impregnated with PA6 were used in various applications, such as the production of organic sheets or core composites with aluminium foam in many thermoplastic manufacturing processes.

\section{Production process}

Uniform samples for bending tests and micrograph analyzes were performed for the investigation of the material properties of the manufactured material composite. The production can be divided into three steps: the preparation, the pressing of the individual layers and the remoulding of the sample plate.

\subsection{Preparation}

First, the unidirectional Prepregs with a PA6 matrix and a fibres volume fraction of $60 \%$ are cut to the appropriate shape with approx. $1 \mathrm{~mm}$ of space per side, so that the fibres in the tool are not compressed. For the samples with fibreglass top layer, three layers per side are used, for the carbon fibre top layers six layers each. In the tool, the core composite is built up in its individual layers (Figure 3). The bottom layer is formed by the Prepregs with fibre orientation along the $\mathrm{x}$-axis, followed by a layer of $100 \mathrm{ml}$ or $85 \mathrm{~g}$ of PA6 granules. The middle layer is $85 \mathrm{ml}$ or $20 \mathrm{~g}$ of expanded glass granules. After that come again a layer PA6 and the upper cover layer.

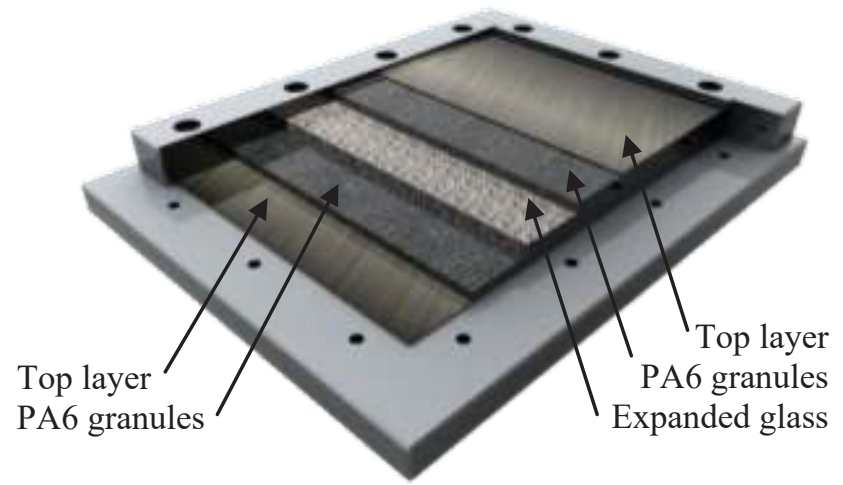

Figure 3. Basic structure of the core composite
In this composition, the core consists of $70 \%$ polyamide and $30 \%$ expanded glass granules. It should be noted that it is the volume fraction in granular form. The bulk density of unreinforced and reinforced PA6 differs only minimally and is negligible due to measurement errors and differences in the individual grains. The threelayered core structure allows the expanded glass granules to be completely enclosed by the plastic matrix. For two samples, each time with glass and carbon fibre as the topcoat, it is examined whether the matrix eventually flows out of the topcoats into the core when the granules for the core are mixed (Figure 4).

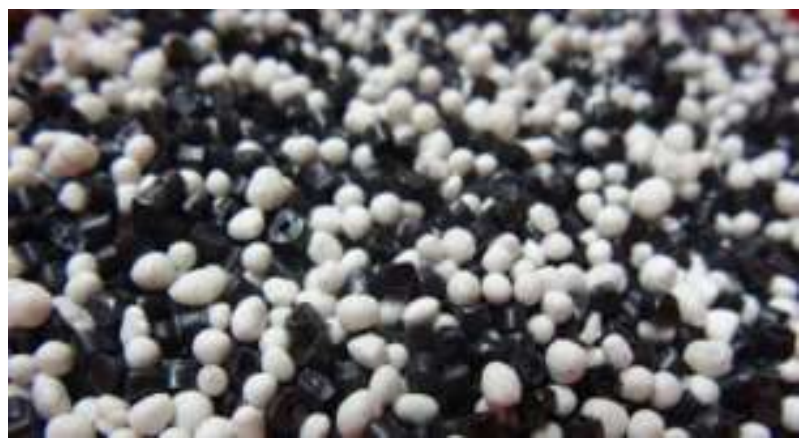

Figure 4. Mixed granules

As a further variant, samples without cover layers are made in order to determine the material properties of the expanded glass granules in conjunction with the PA6. For this, $150 \mathrm{ml}$ or $127.5 \mathrm{~g}$ PA6 are used as the first layer, after which $128 \mathrm{ml}$ or $29 \mathrm{~g}$ expanded glass granules, as well as a further layer PA6. Likewise, samples are made whose cores are composed of a blend ratio of $50 \%$ by volume of PA6 and $50 \%$ by volume of expanded glass granules, and consist entirely of reinforced PA6. For comparison with currently available core materials, samples are also made with aluminium foam.

\subsection{The pressing process}

The production cycle is set on the laboratory press without pressure, because the expanded glass granules are not crushed on the one hand and the second not to be pushed too close to the outer layers. Due to the thermal expansion of the tool and of the plastic, a pressure of approximately $10 \mathrm{~N} / \mathrm{cm}^{2}$ arises after the heating phase.

The cycle time is largely limited by the system-side heating and cooling rate of $15 \mathrm{~K} / \mathrm{min}$ (Figure 5).

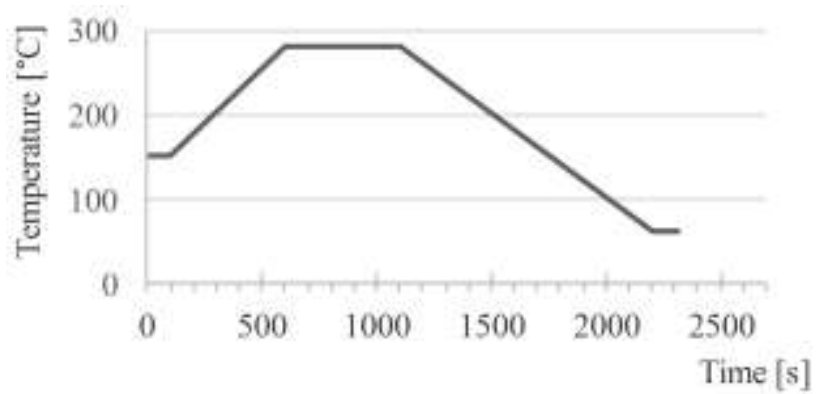

Figure 5. Optimized production cycle 
For the first one hundred seconds, the hotplates are held constant at $150^{\circ} \mathrm{C}$ to bring the tool up to temperature. It is then heated to $280^{\circ} \mathrm{C}$ and held this temperature for 500 s. Afterwards it is cooled to $60^{\circ} \mathrm{C}$. By means of empirical experiments, the machine boundary conditions and the cycle parameters resulting there from during the melting phase are optimized. Overall, there is a cycle time of about 39 minutes.

\subsection{Result}

During the production of varying samples, a direct influence of the starting materials and their composition as well as their mixing ratio and the mixing strategy could be demonstrated. The unreinforced polyamide sank and the expanded glass granulates floated on the melt. It formed very large air pockets. These have a great influence on the material properties.

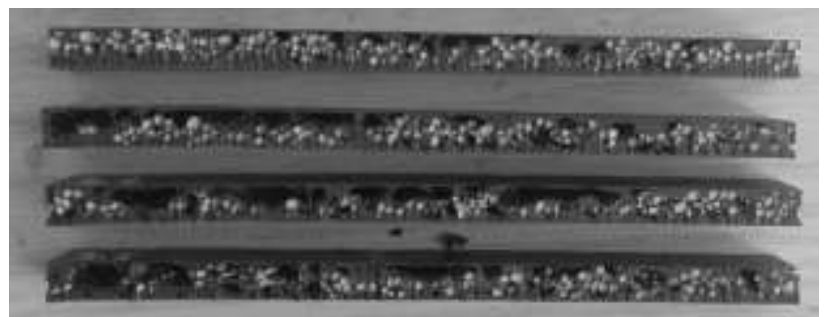

Figure 6. Structure of varying unreinforced samples

In contrast, the reinforced polyamide forms a smallpored, foam-like structure. The fibre reinforcement acts to support and the expanded glass granules are uniformly enclosed by the plastic. Depended on the blend ratios of the reinforced PA6 granulate a varying foam can be adjusted (Figure 7). Based on this knowledge, the samples are made for the investigation and optimization of the mechanical properties with fibre reinforced polyamide. A sample was made with a blend of $50 \%$ expanded glass granules and 50\% glass fiber reinforced polyamide. The specific flexural stiffness in this test is at a level with the 30/70 blend. The higher proportion of expanded glass granules allows a high lightweight construction potential of the core composite and further investigations with different mixing ratios are to be carried out.

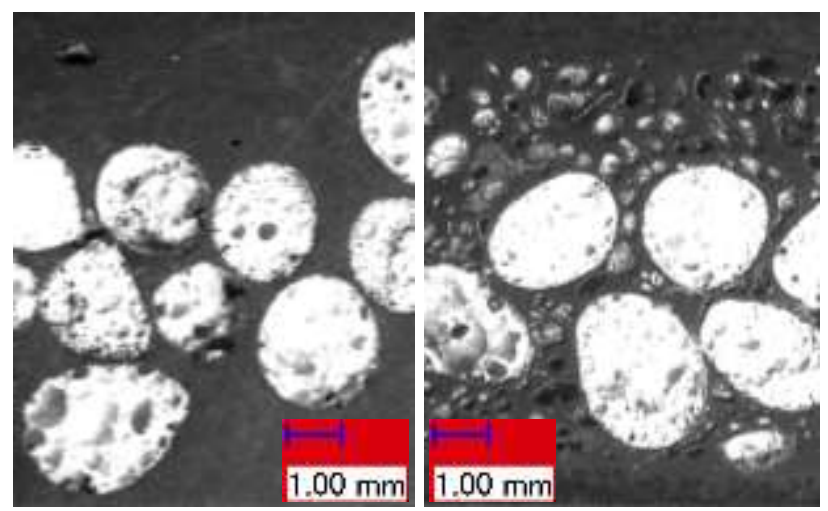

Figure 7. Varying foam samples

A core composite with thermoplastic expanded glass granulate core offers versatile possibilities. Due to the low density of expanded glass granules, it has a high level of lightweight construction potential. Due to the many individual components, there are a variety of combination options, each having different properties. These influencing factors require a closer examination.

\section{Examination of mechanical properties}

There are a total of 17 manufactured plates for 87 samples with the different variant diversity and an unreinforced aluminium foam plate available for the investigation of which specific flexural modulus is determined by means of 4-point bending tests. In addition, 40 cuttings for micrographs are used to analyze the macroscopic as well as the microscopic structure.

\subsection{Bending tests}

The tests are carried out in accordance with DIN 53293 for the testing of core composites. With the 4-point bending test characteristic values of the core composite are to be determined under bending stress. In particular, the bending moment at failure, the tensile and compressive stress in the outer layers, the specific flexural modulus and the specific bending stiffness.

During the bending test, the deflection and the pressing force are recorded. By means of the secant method, by two points in the linear range of the measured values ("Hooke's straight line"), the deflection can be used to calculate the flexural modulus $\mathrm{E}$, where $\Delta \mathrm{F}$ is the change in the pressing force and $\Delta \mathrm{f}$ is the change in the deflection between these points.

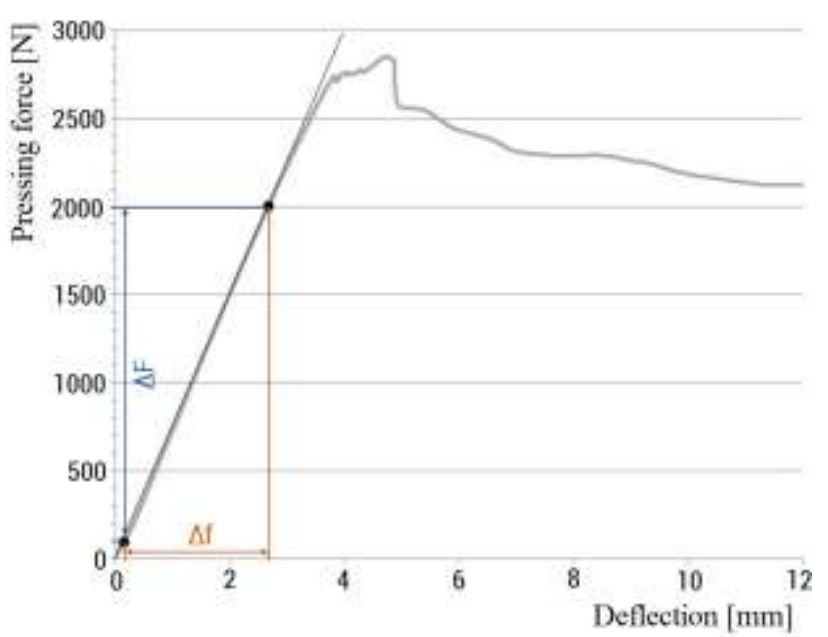

Figure 8. Hooke's straight line (example)

These specific values can now be divided according to the respective core material displayed and analyzed statistically. Here, the region of the second and third as well as the median Quanitl display. The respective largest and smallest measurement is acquired using the error representation. Figure 9 compares the specific bending stiffnes of variants with carbon fibre top layer. 


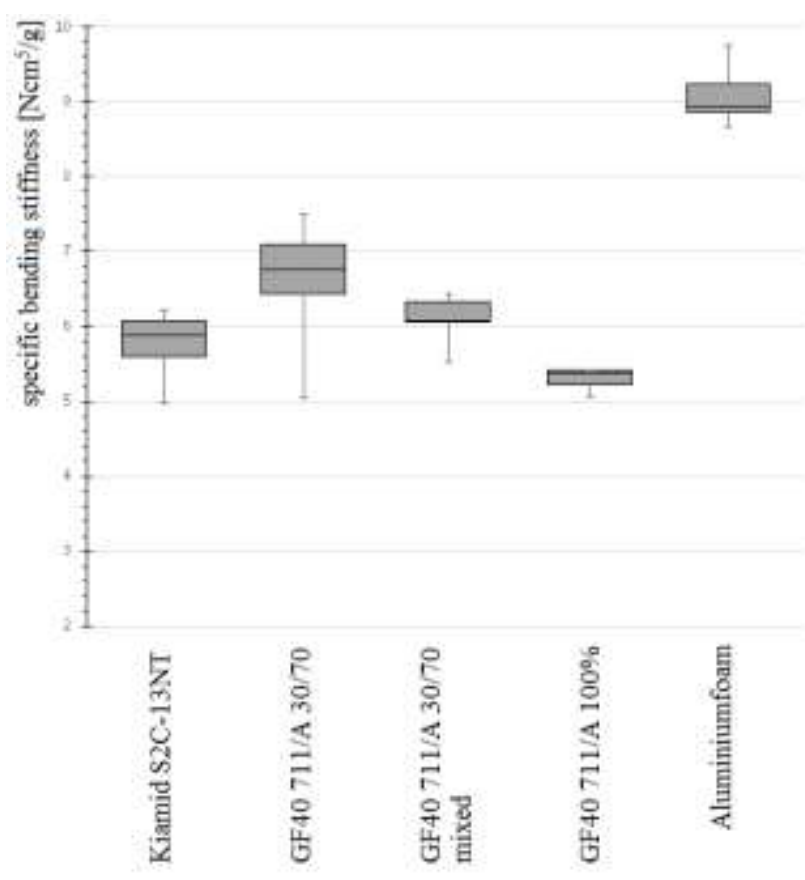

Figure 9. Specific bending stiffness

It turns out that it makes a big difference whether the plastic granulate and the expanded glass granules are applied in layers or mixed directly. However, further investigations are necessary for this. The illustration also shows that the specific bending stiffness of the core without expanded glass granules is significantly lower than all variants with reinforced expanded glass-plastic core. The core values of the mixed granules reach the best values. The unreinforced polymer matrix again turns out to be insufficient. Although the determination of the specific bending stiffness of the samples without cover layer by the 4-point bending test is not universally valid, the individual core materials can be compared with each other. It should be noted that the specific bending strength of the aluminium foam 2.7 times as large as the module of the unreinforced PA6. The reinforced polyamide is approximately in the middle.

\subsection{Micrographs}

In addition to the flexural stiffness tests, many essential properties can also be determined by analyzing micrographs.

For this purpose, the sample plate is cut at a suitable location and the individual blanks embedded in a redcoloured epoxy resin, so that you get a quasi-flat surface. Afterwards, the sample is ground with ever finer sandpaper until the cut surface is exposed. The last step is to polish the surface.

When grinding and polishing, glass particles are always removed from the blown glass grains, so that it is not possible to obtain a completely scratch-free surface. These are visible in the micrographs, but interfere with the analysis only insignificantly. In order to save embedding always several samples are housed in a specific form as shown in Figure 10. The micrographs are viewed under a digital reflected light microscope, with a maximum magnification of 3000x, which allows a direct analysis of the micrographs after recording.

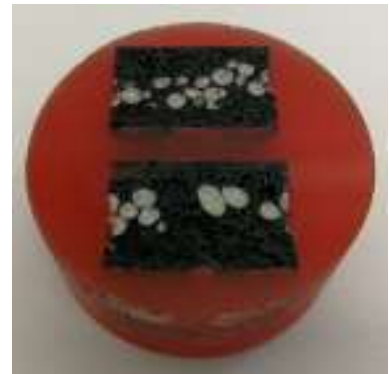

Figure 10. Samples, housed in a specific form

In the micrographs, the following material properties can be examined, which are described in more detail in the following chapter:

- Phase differences and their distribution

- Pore size and distribution

\subsubsection{Phase differences}

In the micrographs, the individual phases or materials can be clearly identified. These are composed of the polyamide matrix, the reinforcing fibres, the expanded glass granules and air inclusions.

Accordingly, the distribution of the expanded glass granules can be analyzed in the macroscopic range. When in samples with unreinforced polyamide as the core material is a "floating" of the expanded glass granules can be seen, with a large part of the granules collected in the upper part of the samples. Also, it can be seen very well on the basis of this micro section, the triggering of the glass particles.

In the samples with reinforced polyamide as the core matrix, a more even distribution of core matrix, expanded glass granules and air inclusions occurs, which explains the lower variability of the material properties in the bending test. In all micrographs, it should be noted positively that the expanded glass granulate is uniformly enclosed by the matrix. Even between closely adjacent granules, a separating layer of matrix material is detectable.

At the boundary layer between the matrix and the expanded glass granules, a dark border form, which at first sight might be interpreted as a gap (see Figure 11). To study this more thoroughly, a depth analysis was performed. With the measuring system of the reflectedlight microscope, a series of images can be created with a small change in the focal length, which are assembled directly into a three-dimensional system in the system.
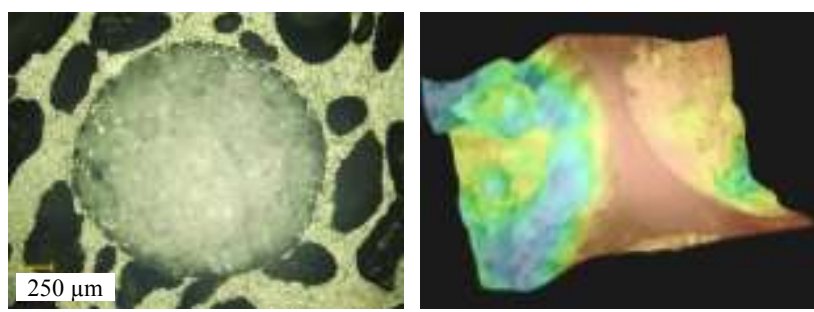

Figure 11. Samples, housed in a specific form 
At the transitions from the cover layer to the matrix material, no phase transition is apparent. The glass fibres of the fibre-reinforced polyamide extend directly to the fibres of the outer layers, but the fibres of the outer layers are clearly visible. The missing boundary layer shows that the cover layers are optimally connected to the core.

\subsubsection{Pore size and distribution}

In the unreinforced polyamide core large air pockets occur, which are distributed irregularly. An analysis is therefore not possible. A closer examination is only possible with the samples with reinforced core, which can also be performed directly with the measuring system of the microscope. Macroscopically, these samples show a uniform distribution of the pores. The evaluation is carried out in areas without expanded glass granulates. On the one hand, the diameter of the pores can be measured directly. On average, over six micrographs and 332 measuring sites, the mean pore diameter is $185 \mu \mathrm{m}$ at the cutting planes. However, since this is a section through a three-dimensional structure, these values are not very meaningful individually. The average, maximum pore diameter is probably much larger. More meaningful is the comparison of the pore area to the total area. Assuming that the pores are randomly distributed throughout the core and extend evenly in all three dimensions, quantitative statements can be made. For this analysis, the micrograph is decolorized and divided into two areas, black and white. From these areas, the respective area can be determined and compared. This procedure is shown in Figure 12.

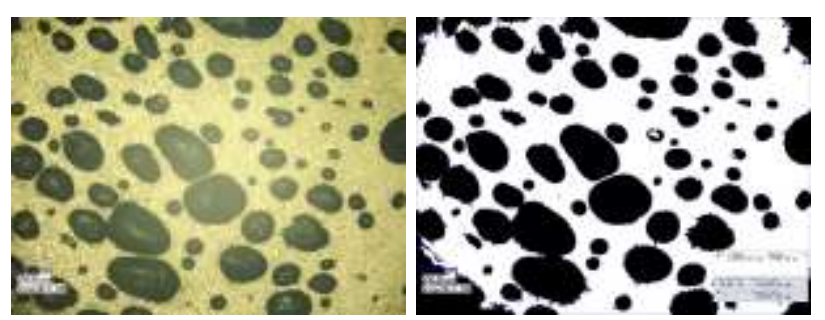

Figure 12. Surface analysis of a micrograph

After analyzing 8 micrographs, the average ratio is $44 \%$ matrix and $65 \%$ pores. If one deducts the deviations in the border areas one arrives approximately on a relationship of $50 \%$ matrix to $50 \%$ air inclusions. In addition to the low density of expanded glass granules, this brings another important factor in the weight savings of the core composite.

\section{Summary}

Compared with core materials currently available in the prior art, the core composite with thermoplastic glass fibre reinforced expanded glass granule core has similar mechanical properties. It should be emphasized positively that the cover layers are firmly bonded to the core, which makes delamination in the boundary region between the cover layer and the core difficult. In 4-point bending, most reinforced core samples fail due to the shear forces in the core. The characteristic of this failure is a $45^{\circ}$ inclined to the surface fracture. Occasionally it occurred in the bending samples with unreinforced PA6 to the top layer break at the force application points. This may be due to a higher toughness of the core material. Due to the fibre reinforcement, the core is much stiffer and can dissipate much less energy via plastic deformation. This is especially negative for crash structures.

It should also be noted that expanded glass granules in the vicinity of the cover layers can change the thickness of these. However, it has to be further investigated whether these negatively affect the properties. Since the fibres in the cover layers are continuous fibres, they are only shifted, but not interrupted, and can pass on the tensile forces unhindered. At pressure stress, this deformation can already lead to failure, however, further investigations are necessary because any support effects relatives the buckling of the fibres again.

Due to the large variation of the specific bending stiffness in the samples with reinforced polyamide, further bending tests must be carried out in order to be able to make more precise statements. The individual constituents of the core material were measured by weight. Due to different shapes and granule sizes, however, it will make more sense in the future to carry out the dimensioning by volume. For this a suitable procedure has to be found.

\section{Acknowledgement}

These studies were performed as part of a preinvestigation within the Federal Cluster of Excellence EXC 1075 "MERGE Technologies for Multifunctional Lightweight Structures" and supported by the German Research Foundation (DFG) to explore integrative inmold processes with plastic / metal composites. Financial support is gratefully acknowledged.

\section{References}

1. Hackert, A.; Timmel, T.; Drebenstedt, C.; Osiecki, T.; Kroll, L.: Composite Sandwich with Aluminium Foam core and adhesive bonded Carbon Fibre Reinforced Thermoplastic cover layer. In: Key Engineering Materials 2017, No.744, pp. 277-281 DOI: 10.4028/www.scientific.net/KEM.744, ISBN13: 978-3-0357-1116-5

2. Nestler, D. J.: Beitrag zum Thema Verbundwerkstoffe - Werkstoffverbunde. Status quo und Forschungsansätze, Habilitation, Technische Universität Chemnitz, 2012.

3. Hackert, A.; Müller, S.; Kroll, L.: Lightweight Wheel Disc with Carbon Aluminium Foam Sandwich. Lightweight Design worldwide, March 2017, Volume 10, Issue 1, pp. 6-11, DOI: 10.1007/s41777017-0016-2, ISSN 2510-2877

4. Osiecki, T.; Gerstenberger, C.; Hackert, A.; Seidlitz, H.; Kroll, L.: Thermoplastic Fiber Reinforced/MetalHybrid Laminates for Structural Lightweight Applications. 23rd Annual International Conference on Composites/Nano Engineering (ICCE-23). Chengdu (China), 2015. 